\title{
Optimizing propagating spin wave spectroscopy ${ }^{\oplus}$
}

Cite as: Appl. Phys. Lett. 115, 012403 (2019); https://doi.org/10.1063/1.5090892

Submitted: 30 January 2019 . Accepted: 16 June 2019 . Published Online: 02 July 2019

Juriaan Lucassen, Casper F. Schippers (D), Luuk Rutten, Rembert A. Duine, Henk J. M. Swagten, Bert Koopmans (D), and Reinoud Lavrijsen (i)

\section{COLLECTIONS}

This paper was selected as an Editor's Pick
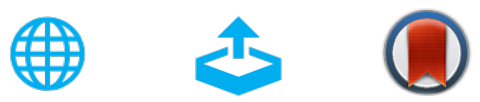

\section{ARTICLES YOU MAY BE INTERESTED IN}

Structural, magnetic, and electrical properties of collinear antiferromagnetic heteroepitaxy cubic $\mathrm{Mn}_{3} \mathrm{Ga}$ thin films

Applied Physics Letters 115, 012402 (2019); https://doi.org/10.1063/1.5088790

A correlation between electron-hole pair radii and magnetomodulation of exciplex

fluorescence in electron donor-electron acceptor organic systems

Applied Physics Letters 115, 013301 (2019); https://doi.org/10.1063/1.5095878

Spin-current diode with a monoaxial chiral magnet

Applied Physics Letters 115, 012401 (2019); https://doi.org/10.1063/1.5097866

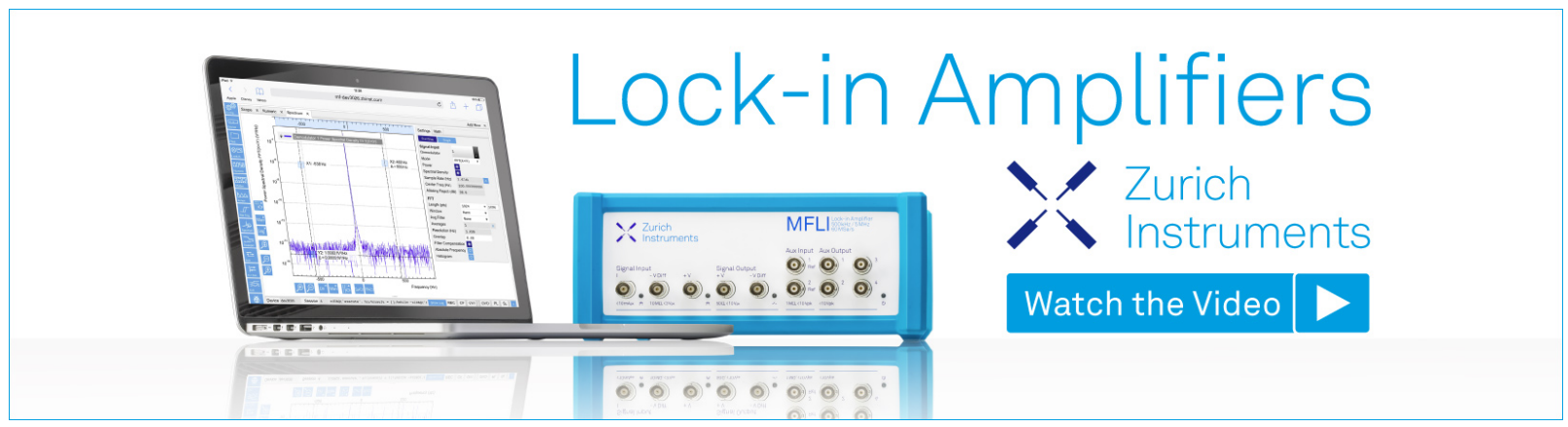




\title{
Optimizing propagating spin wave spectroscopy 루
}

Cite as: Appl. Phys. Lett. 115, 012403 (2019); doi: 10.1063/1.5090892

Submitted: 30 January 2019 . Accepted: 16 June 2019 .

Published Online: 2 July 2019

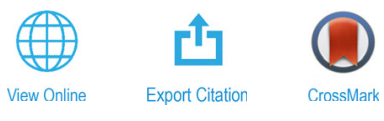

Juriaan Lucassen, ${ }^{1, \text { a) }}$ Casper F. Schippers, ${ }^{1}$ (D) Luuk Rutten, ${ }^{7}$ Rembert A. Duine, ${ }^{1,2}$ Henk J. M. Swagten, ${ }^{1}$ Bert Koopmans, ' iD and Reinoud Lavrijsen (iD

\author{
AFFILIATIONS \\ 'Department of Applied Physics, Eindhoven University of Technology, P.O. Box 513, 5600 MB Eindhoven, The Netherlands \\ ${ }^{2}$ Institute for Theoretical Physics, Utrecht University, Princetonplein 5, 3584 CC Utrecht, The Netherlands
}

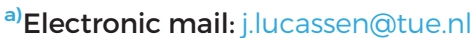

\begin{abstract}
The frequency difference between two oppositely propagating spin waves can be used to probe several interesting magnetic properties, such as the Dzyaloshinskii-Moriya interaction (DMI). Propagating spin wave spectroscopy is a technique that is very sensitive to this frequency difference. Here, we show several elements that are important to optimize devices for such a measurement. We demonstrate that for wide magnetic strips, there is a need for de-embedding. Additionally, for these wide strips, there is a large parasitic antenna-antenna coupling that obfuscates any spin wave transmission signal, which is remedied by moving to smaller strips. The conventional antenna design excites spin waves with two different wave vectors. As the magnetic layers become thinner, the resulting resonances move closer together and become very difficult to disentangle. In the last part, we therefore propose and verify an alternative antenna design that excites spin waves with only one wave vector. We suggest to use this antenna design to quantify the DMI in thin magnetic layers.
\end{abstract}

Published under license by AIP Publishing. https://doi.org/10.1063/1.5090892

Spin waves can be used to probe fundamental magnetic interactions in a ferromagnet. For example, the uniform spin wave mode is routinely used to determine the magnetic anisotropy in ferromagnetic resonance based techniques. ${ }^{1,2}$ More recent advances have demonstrated that spin waves can further be used to probe spin polarized transport $^{3-5}$ and they are also frequently used to quantify the Dzyaloshinskii-Moriya interaction (DMI). ${ }^{6-9}$ The use of spin waves to quantify the DMI is especially interesting because the field of skyrmionics revolves around this DMI. ${ }^{10}$ Spin waves are one of the few ways of quantifying this interaction. ${ }^{6-9,11-13}$ Quantifying the DMI using spin waves utilizes the frequency difference for oppositely propagating spin waves as a direct result of the DMI. ${ }^{14,15}$ The most commonly used method to measure this frequency difference is Brillouin light scattering. ${ }^{7-9}$

Here, we focus on the related, though less developed, technique of propagating spin wave spectroscopy (PSWS) ${ }^{3}$ that can also measure the DMI induced frequency difference. ${ }^{6,16}$ In PSWS, a micrometer sized coplanar waveguide (CPW) is used to electrically generate spin waves with a specific wavevector in a magnetic strip via Oersted fields. These spin waves propagate toward a second antenna, where the spin waves are detected inductively. Although in principle, PSWS is very sensitive to frequency differences, the fabrication of the devices is involved, and important details that are critical to correct operation remain underreported.
In this letter, we demonstrate that the width of the magnetic strip critically determines the functionality of the device, with narrow strips being optimal. First, we show that correcting for the finite length of the microwave contacts (de-embedding) becomes important as the strip width increases. Second, for narrow strips, the spectra show additional resonances that belong to spin wave quantization modes along the strip width. Third, upon increasing the strip width, we additionally find that the antenna-antenna coupling also increases, which detrimentally affects the spin wave transmission measurements. Finally, we show an alternative antenna design which does not excite a second spin wave resonance. This should aid in the determination of DMI in magnetic films as it allows the measurements to be performed for decreased strip thicknesses where the DMI is higher. Moreover, magnonic applications that require the presence of more monochromatic spin waves can also benefit from this design.

We fabricated devices such as the one displayed in Fig. 1(a). The operating principle of such a device is described in detail elsewhere. ${ }^{17}$ In short, as we indicate in red in the figure, we drive a microwave current $j$ through one of the antennas. The spatial periodicity of the Oersted fields that couple to the spin waves is determined by the geometry of the antenna. Because there are two main periodicities, indicated by $k_{\mathrm{m}}$ and $k_{\mathrm{s}}$ in the figure, we also excite spin waves with these wave vectors. Spin waves then traverse the strip to the other antenna, where induction allows the spin waves to be detected. The 

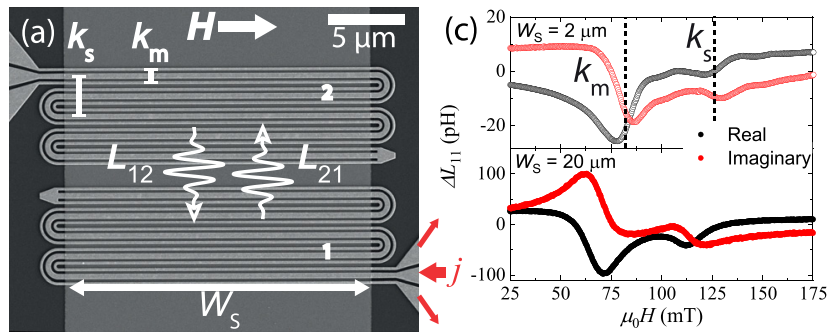

(b)

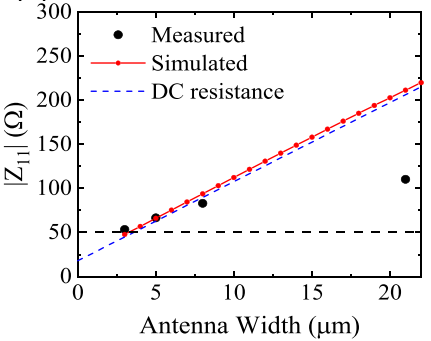

(d)

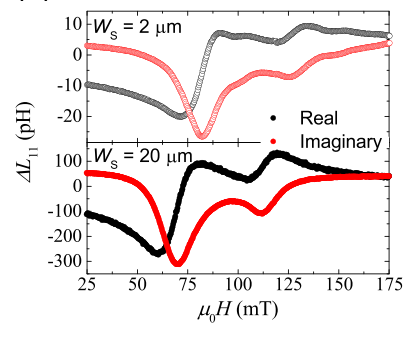

FIG. 1. (a) SEM micrograph of a fabricated device with $k_{\mathrm{m}}=7 \mu \mathrm{m}^{-1}$ for a strip width $W_{S}$ of $20 \mu \mathrm{m}$. Also indicated are the direction of the magnetic field $H$, alternating current (j) flow directions, main periodicities of the antenna, and antenna number. $L_{x y}$ indicates the spin wave flow direction and corresponding mutual induction. (b) Measured absolute antenna impedances $Z_{11}$ as a function of the antenna width at $14 \mathrm{GHz}$. Plotted together with the simulated antenna impedances as well as the calculated theoretical DC resistance of the antenna (see the supplementary material). The dashed horizontal line indicates $Z_{0}=50 \Omega$. (c) Raw $\Delta L_{11}$ data at $14 \mathrm{GHz}$ with $k_{\mathrm{m}}=7 \mu \mathrm{m}^{-1}$ for $W_{\mathrm{S}}=2 \mathrm{~m}$ (top) and $20 \mu \mathrm{m}$ (bottom). The two peaks, indicated by the dashed lines, correspond to the main periodicities of the antenna [see (a)]. (d) De-embedded version of data shown in (c).

magnetic strip underneath the antenna is fabricated using DC magnetron sputter deposition and an electron beam lithography (EBL) liftoff process. The sputtered stack is $/ / \mathrm{Ta}(4) / \mathrm{Pt}(4) / \mathrm{Co}(15) / \operatorname{Ir}(4) / \mathrm{Pt}(4)$ (numbers in parentheses are thicknesses in nanometers) and was deposited using Ar at $1 \times 10^{-2}$ mbar on a Si substrate with a native oxide in a system with a base pressure of $2 \times 10^{-9} \mathrm{mbar}$. On top of the magnetic strip, we deposited $40 \mathrm{~nm}$ of $\mathrm{Al}_{2} \mathrm{O}_{3}$ using atomic layer deposition. Finally, the antennas were created using e-beam evaporation of $\mathrm{Ti}(10) / \mathrm{Au}(100)$ in a second EBL lift-off process. The following geometrical parameters were used for the antenna design: ground line width $l_{\mathrm{p}}=\frac{2 \pi}{5 k_{\mathrm{m}}}$, signal linewidth $2 l_{\mathrm{p}}$, ground-ground spacing $\frac{3}{2} l_{\mathrm{p}}$, and signal-ground spacing $l_{\mathrm{p}}$. ${ }^{17} \mathrm{We}$ performed the spin wave resonance measurements using an Anritsu MS4644B Vector Network Analyzer (VNA) which we brought into contact with the antennas using microwave probes. The VNA was calibrated using a microwave probe calibration substrate. Measurements were performed in the field sweep mode with the magnetic field $H$ applied transverse to the strip, working in the Damon-Eshbach geometry at a power of $0 \mathrm{dBm}$. Afterwards, the measured $S$ parameters were converted to inductions using wellknown microwave relationships. ${ }^{18}$ Devices were fabricated for various strip widths $W_{\mathrm{S}}(2-20 \mu \mathrm{m}$, where the antenna width includes an additional $0.5 \mu \mathrm{m}$ on each side) and antennas that were designed to excite different wave vectors $\left(k_{\mathrm{m}}=5-9 \mu \mathrm{m}^{-1}\right)$.

We start by looking at a typical measurement of the selfinduction $\Delta L_{11}$ as shown in Fig. 1 (c) for two different $W_{\mathrm{S}}$ values. These measurements correspond to the amplitude of the spin waves that are excited by antenna 1 . We note two different peaks, indicated by the dashed lines, at two different fields which correspond to the two $\left(k_{\mathrm{m}}\right.$ and $\left.k_{\mathrm{s}}\right)$ wave vectors of spin waves that are excited (later verified by fitting the dispersion relation). Additionally, the curves resemble the (anti)symmetric Lorentzian line shapes typical of ferromagnetic resonance for both strip widths. The phase for $W_{\mathrm{S}}=2 \mu \mathrm{m}$ matches what one would expect for magnetic resonance: a symmetric imaginary induction and antisymmetric real induction. ${ }^{2}$ However, the phase of the $W_{\mathrm{S}}=20 \mu \mathrm{m}$ device behaves rather differently, where the roles of the real and imaginary parts are now interchanged.

In Fig. 1(d), we plot the measurements of the self-induction corrected for the small change in the phase of the $S$ parameters as a result of the finite distance between the probes and the actual spin wave antenna. This process is called de-embedding. ${ }^{18}$ For $W_{\mathrm{S}}=2 \mu \mathrm{m}$, there is very little effect of de-embedding. However, for $W_{\mathrm{S}}=20 \mu \mathrm{m}$, the phase of the spin wave resonances changes drastically and now matches the $W_{S}=2 \mu \mathrm{m}$ data. This is a rather surprising result because the induced phase difference $\theta$ as a result of the finite distance is only $\sim 40^{\circ}$ at $15 \mathrm{GHz}$. Additionally, de-embedding only seems to be important for wider strips. To understand this behavior, we derive the following relationship (with $\theta \ll 1$ ) for a 1-port circuit ${ }^{18}$

$$
\Delta L_{11} \rightarrow \Delta L_{11}^{*}\left(1+\frac{i \theta Z_{11}}{Z_{0}}\right),
$$

where $\Delta L_{11}$ is the proper de-embedded self-induction and $\Delta L_{11}^{*}$ the measured self-induction. $Z_{11}$ is the nonmagnetic part of the impedance of the antenna, and $Z_{0}$ is the characteristic impedance of the line (50 $\Omega$ ). This was derived with the total antenna taken as a terminating impedance (with impedance $Z_{11}+\Delta L_{11}$ ) of the line. From this, it is clear that de-embedding becomes more important as $Z_{11}$ increases. In Fig. 1(b), $\left|Z_{11}\right|$ is plotted as a function of antenna width. It increases linearly with the antenna width, which explains why there is a much larger effect of de-embedding for larger $W_{\mathrm{S}}$. This linear increase can be understood very simply in terms of the DC resistance of the antenna, which dominates the inductance and capacitance of the antennas because the conduction lines are very resistive due to their small size (see the supplementary material).

Additionally, we see in Fig. 1(c) that the magnitude of the induction is only about 5 times larger for $W_{\mathrm{S}}=20 \mu \mathrm{m}$ compared to $W_{\mathrm{S}}=2 \mu \mathrm{m}$. The induction should scale linearly with the magnetic volume, which is exactly what is found in Fig. 1(d): a tenfold increase in the induction going from the $2 \mu \mathrm{m}$ strip to the $20 \mu \mathrm{m}$ strip. Once again, this can be understood from Eq. (1); there is not only a phase rotation present but also a multiplicative term proportional to $Z_{11}$. Although moving to smaller $W_{\mathrm{S}}$ will help decrease $Z_{11}$ and thus remove the need for de-embedding, something similar can be achieved by decreasing the resistance of the antenna. For example, one can increase the thickness of antenna. ${ }^{19}$

Next, we demonstrate that upon decreasing $W_{S}$, a spin wave quantization resonance appears in the spectra. To see this more clearly, we plot $L_{11}$ data for a $W_{\mathrm{S}}=2 \mu \mathrm{m}$ strip in Fig. 2(a). Once again note that there are two main peaks present in this figure, the $k_{\mathrm{m}}$ peak at $\sim 110 \mathrm{mT}$ and the $k_{\mathrm{s}}$ peak at $\sim 150 \mathrm{mT}$, but there is clearly another resonance visible at $\sim 140 \mathrm{mT}$. This resonance vanishes as $W_{\mathrm{S}}$ is increased to $20 \mu \mathrm{m}$. From this, we conclude that any additional periodicities of the antenna geometry that can couple to this spin wave can be excluded because then it should be present for both $W_{\mathrm{S}}=2$ and $20 \mu \mathrm{m}$ devices. ${ }^{17}$ Instead, we believe it to be a higher order laterally 
(a)

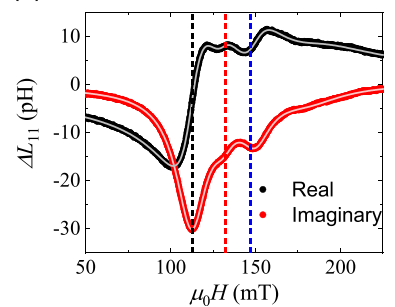

(b)

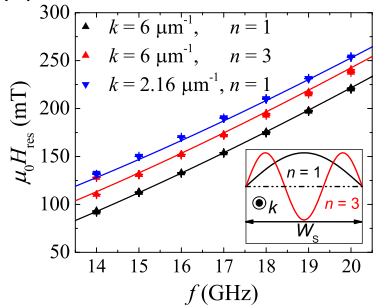

FIG. 2. Data with $k_{\mathrm{m}}=6 \mu \mathrm{m}^{-1}$ at $W_{\mathrm{S}}=2 \mu \mathrm{m}$. (a) $\Delta \mathrm{L}_{11}$ at $15 \mathrm{GHz}$ plotted together with a fit of the 3 spin wave resonances observed. The vertical dashed lines indicate the resonance fields $H_{\text {res }}$ obtained from the fit. (b) Fitted resonance fields $H_{\text {res }}$ as a function of frequency $f$ [see (a)]. In the inset, we show schematically the lateral (along the strip width) spin wave quantization modes that are used to fit the resonance fields.

quantized spin wave mode [inset Fig. 2(b)], which is one of the few reports of spin wave quantization measured using PSWS. ${ }^{20}$

A more detailed quantitative analysis can be performed by fitting the dispersion relation to the resonance fields $H_{\text {res }}$. We obtain these $H_{\text {res }}$ values by fitting the spectrum of Fig. 2(a) to a combination of symmetric and antisymmetric Lorentzian lineshapes. ${ }^{21}$ The resulting $H_{\text {res }}$ values are indicated by the vertical dashed lines. For all three resonances, $H_{\text {res }}$ is plotted in Fig. 2(b) as a function of the frequency $f$.

These curves are fitted simultaneously using dispersion relations derived elsewhere, ${ }^{22,23}$ which are also plotted in Fig. 2(b). Here, we use $g=2.17, M_{\mathrm{s}}=1.44 \mathrm{MA} \mathrm{m}^{-1}$, and $k_{\mathrm{s}, \mathrm{m}}=2.16,6 \mu \mathrm{m}^{-1}$ (fixed by the antenna geometry). We assume that the quantized spin wave mode is an $n=3$ mode [mode profiles are indicated in the inset of Fig. 2(b)] because the excitation efficiency for the $n=2$ mode is negligible. ${ }^{24}$ The quantization is taken into account by adding a wavevector $k=\frac{n \pi}{W_{\mathrm{s}}}$ perpendicular to the propagation direction in the dispersion relation. ${ }^{25}$ We use the following fit parameters: an effective strip width $w_{\text {eff }}$, layer thickness $t$, and $M_{\text {eff }}=M_{\mathrm{s}}-H_{\mathrm{K}}$, with $H_{\mathrm{K}}$ being the magnetic anisotropy field. The resulting fit gives $M_{\mathrm{eff}}=1.1 \pm 0.1 \mathrm{MA} \mathrm{m}{ }^{-1}$, $w_{\text {eff }}=1.1 \pm 0.8 \mu \mathrm{m}$, and $t=11 \pm 4 \mathrm{~nm}$. $M_{\text {eff }}$ is reasonable for this system. ${ }^{6}$ Because we do not take into account the nonuniform internal dipolar fields, ${ }^{25,26}$ the underestimation of $W_{\mathrm{S}}$ and $t$ is not surprising. In the supplementary material, we present fits for devices with different $k_{\mathrm{m}}$ values.

We now turn our attention to the spin wave transmission measurements. A typical measurement for $W_{\mathrm{S}}=2 \mu \mathrm{m}$ is plotted in Fig. 3(a), where we plot the mutual induction $\Delta L_{12}\left(\Delta L_{21}\right)$ which corresponds to spin waves traveling from antennas 2 (1) to 1 (2) [see Fig. $1(\mathrm{a})$. Once again, we can distinguish two peaks corresponding to the two different types of spin waves that are excited. Note two very distinct features that are indicative of a proper electrical spin wave transmission signal: first, a distinct amplitude asymmetry between oppositely traveling spin waves $\left(L_{12}\right.$ vs $\left.L_{21}\right)$, which is the result of the chirality of the driving fields that match the corresponding spin wave $\left(L_{12}\right)$ or oppose it $\left(L_{21}\right) ;$ in second, sharp oscillations of the spin wave transmission signal, which are the result of a variation in the spin wave phase as we sweep through the resonance. ${ }^{17}$

However, a similar measurement for $W_{\mathrm{S}}=20 \mu \mathrm{m}$ is shown in Fig. 3(b). Both the amplitude asymmetry and the sharp oscillations now no longer seem to be present. This is rather surprising as both (a)

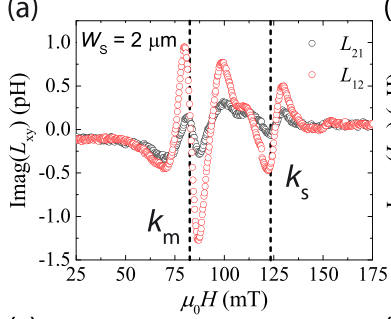

(b)
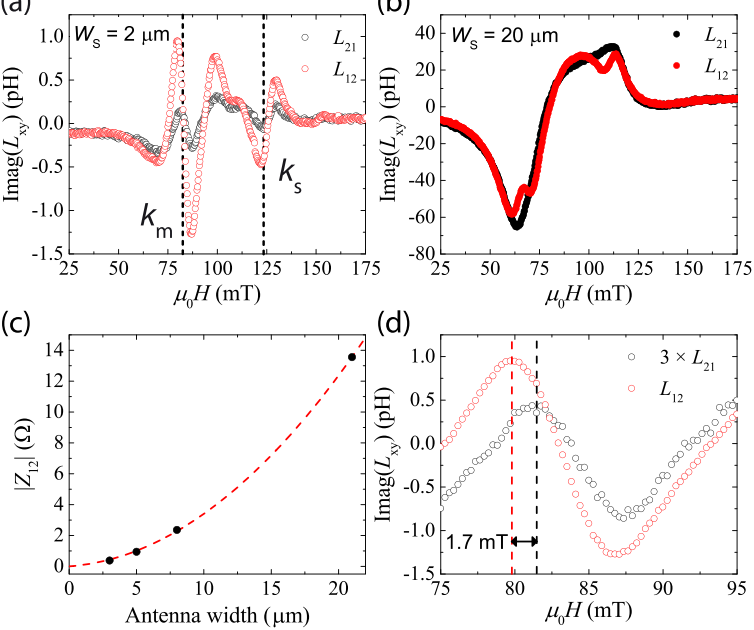

(d)

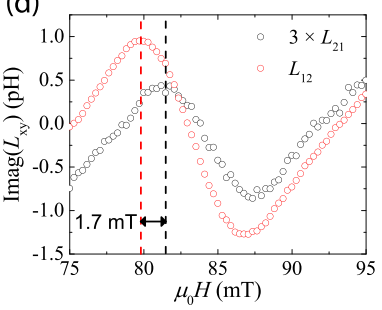

FIG. 3. $\operatorname{Imag}\left(\Delta L_{\mathrm{xy}}\right)$ data at $14 \mathrm{GHz}$ with $k_{\mathrm{m}}=7 \mu \mathrm{m}^{-1}$ for $W_{\mathrm{S}}=2 \mu \mathrm{m}$ (a) and $20 \mu \mathrm{m}$ (b). The two dashed lines in (a) indicate the resonance fields of spin waves corresponding to the main periodicities of the antenna [see Fig. 1(a)]. (c) Absolute value of the antenna-antenna coupling impedance $Z_{12}$ as a function of the antenna width. The line is a guide to the eye, based on a quadratic fit. (d) Zoomed-in version of (a) with the peak shift of $\sim 1.7 \mathrm{mT}$ indicated. Here, $L_{21}$ was artificially blown up to make the peak shift easier to see.

features have an origin that does not depend on $W_{\mathrm{S}}$. Rather, we believe that it is related to a direct parasitic coupling between the two antennas. This means that if a spin wave is excited by antenna 1, there is a signal induced in antenna 2 independent of an actual physical spin wave being transmitted. ${ }^{28}$ For example, for $L_{12}$, there is still a small oscillatory signal superimposed on the large resonant background. This background is the result of the parasitic coupling, and the small superimposed signal is the transmitted spin wave. The spin wave transmission signal for $L_{21}$ is smaller, as observed in Fig. 3(a), such that the smaller oscillatory signal on top of this induction is no longer visible in Fig. 3(b).

The magnitude of the parasitic coupling $\left|Z_{12}\right|$ is plotted in Fig. 3 (c), where we find that the coupling seems to scale quadratically with the antenna width. This explains why devices with smaller $W_{\mathrm{S}}$ do show a proper spin wave transmission signal. Yet, even for small $W_{\mathrm{S}}$, this parasitic coupling can become problematic at higher frequencies, where the increasing spin wave attenuation decreases the spin wave transmission signal. ${ }^{29}$ At present, we cannot explain the size and behavior of this coupling, but more details can be found in the supplementary material. Furthermore, additional self-induction and spin wave transmission measurements on devices with different widths $W_{\mathrm{S}}$ between 2 and $20 \mu \mathrm{m}$ can be found in the supplementary material.

For the $W_{\mathrm{S}}=2 \mu \mathrm{m}$ device, a peak shift can be extracted that could be a measure for the DMI. This shift is shown in Fig. 3(d), where $L_{12}$ is shifted about $+1.7 \mathrm{mT}$ with respect to $L_{21}$. The shift is opposite to the direction expected from DMI [assuming $D_{\mathrm{s}}=1.8 \mathrm{pJ} \mathrm{m}^{-1}$ (Ref. 13)], which is about $-1.7 \mathrm{mT}$. This shift can have other contributions beyond the DMI, such as the anisotropy difference induced shift. ${ }^{30}$ Upon moving to thinner layers, this contribution should decrease in size, and the contribution of the DMI to the field shift will increase. Therefore, in future work, we would like to investigate thinner layers to determine the origin of this shift. 
In the final part of this letter, we present an alternative antenna design. This has the major advantage of exciting only one type of spin wave $\left(k_{\mathrm{m}}\right)$ which is necessary if thinner layers have to be investigated. Upon decreasing the layer thickness, the two traditional spin wave resonances (corresponding to $k_{\mathrm{m}}$ and $k_{\mathrm{s}}$ ) start overlapping because of the decreasing influence of the magnetostatic interactions (see the supplementary material for a more detailed investigation). Although the ratio between the $k_{\mathrm{m}}$ and $k_{\mathrm{s}}$ resonance is quite large for $L_{11}$, they are of approximately equal size in the transmission measurement as seen in Fig. 3(a). If the two peaks move closer together, disentangling the two resonances becomes increasingly difficult in a transmission measurement.

The alternative antenna design is shown in Fig. 4(a). Rather than relying on a conventional CPW signal and ground line structure, in this alternative design, only the signal line is meandered. There is no need to adhere to conventional CPW structures for these spin wave antennas as the antennas are much smaller than the electrical wavelength. In the figure, we indicate the only periodicity $k_{\mathrm{m}}$ present such that spin waves with only one wave vector are excited. This should negate the problem of overlapping spin wave resonances in the transmission induction spectra. To further illustrate how this works, note that in Ref. 17, it is demonstrated that the spin wave excitation signals are proportional to the square of the spatial Fourier transform of the current density used to excite the spin waves. In Fig. 4(b), the Fourier transforms of the current density for both the conventional [Fig. 1(a)] and alternative [Fig. 4(a)] antenna designs are plotted. For the conventional design, there are two peaks $\left(k_{\mathrm{s}}\right.$ and $\left.k_{\mathrm{m}}\right)$ that correspond to the
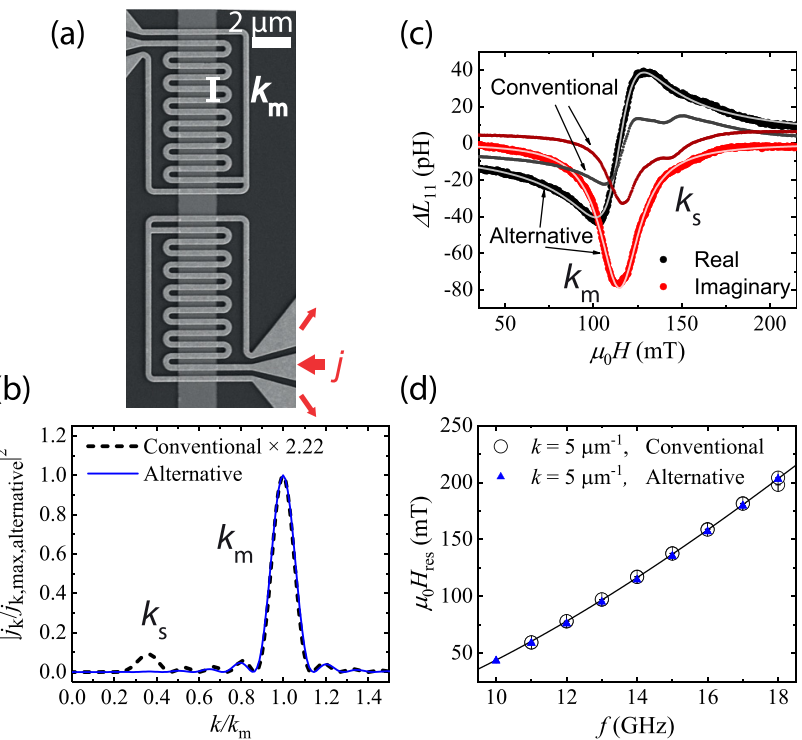

(d)

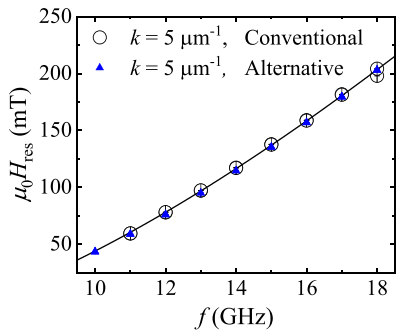

FIG. 4. (a) SEM micrograph of a fabricated device with $k_{\mathrm{m}}=5 \mu \mathrm{m}^{-1}$ and $W_{\mathrm{S}}=2 \mu \mathrm{m}$. Also indicated are the alternating current (j) flow directions, and main periodicity of the antenna. (b) Square of the Fourier transform of the current density $j_{k}$ of the conventional and alternative antenna design. Notice the scaling of the conventional antenna design. (c) $\Delta L_{11}$ at $14 \mathrm{GHz}$ with $k_{\mathrm{m}}=5 \mu \mathrm{m}^{-1}$ for $W_{\mathrm{S}}=2 \mu \mathrm{m}$ for both conventional and alternative antenna designs. For the alternative design, the solid line is the result of a fit. (d) Resonance fields $H_{\text {res }}$ as a function of frequency $f$ at $k_{\mathrm{m}}=5 \mu \mathrm{m}^{-1}$ and $n=1$ for both the conventional and alternative design. The fit belongs to the complete dataset of the conventional design. two spin wave resonances that are measured. For the alternative design, however, the secondary peak at $k_{\mathrm{s}}$ disappears, meaning that with this alternative design, spin waves with only one wave vector $k_{\mathrm{m}}$ are excited. Moreover, the $k_{\mathrm{m}}$ peak of the alternative design is $\sim 2$ times larger than the conventional design as a result of the higher current density that flows through the alternative design, suggesting that the induction signals should also be larger.

We verify these predictions by measuring the self-induction $\Delta L_{11}$ for the alternative antenna design; this is plotted in Fig. 4(c) together with a similar measurement on a device with the conventional CPWbased antenna design. As can be seen, the secondary peak at $k_{\mathrm{s}}$ has vanished for the alternative antenna design, agreeing with our initial expectations based on the periodicity of the antenna. The intensity of the signal is also a factor $\sim 2$ larger which agrees with the initial predictions based on the current density.

A more thorough analysis is obtained by fitting the spectra to obtain the resonance fields $H_{\text {res. }}$. Such a fit is also displayed in Fig. 4(c) with solid lines. ${ }^{32}$ Combining this with a dispersion relation analysis similar to the one performed in Fig. 2(b) yields Fig. 4(d), where we plot only the main resonance field of the spectra. The resonance fields for the alternative design, shown in blue, lay perfectly on top of the data of the conventional design. ${ }^{31}$ In the supplementary material, we show a typical spin wave transmission measurement for this alternative design, and we additionally demonstrate that the parasitic coupling for this design is about a factor of 2 larger compared to the conventional design.

In summary, we have demonstrated the benefit of using narrower strips for propagating spin wave spectroscopy (PSWS). We ended the letter with a demonstration of an alternative antenna design that allowed us to excite spin waves with only one wave vector suitable for the investigation of DMI in thinner films.

See the supplementary material for (1) details on the COMSOL $^{\mathrm{TM}}$ simulations, (2) additional $Z_{11}$ information, (3) a full dispersion relation fit, (4) a set of data for different strip widths, (5) additional details on the parasitic coupling $Z_{21}$, (6) calculations that demonstrate the overlapping of the resonances, and (7) details on the transmission of the alternative design.

This work is part of the research programme of the Foundation for Fundamental Research on Matter (FOM), which is part of the Netherlands Organisation for Scientific Research (NWO).

\section{REFERENCES}

${ }^{1}$ C. Kittel, Phys. Rev. 73, 155 (1948).

${ }^{2}$ I. S. Maksymov and M. Kostylev, Phys. E 69, 253 (2015).

${ }^{3}$ V. Vlaminck and M. Bailleul, Science 322, 410 (2008).

${ }^{4}$ M. Zhu, C. L. Dennis, and R. D. McMichael, Phys. Rev. B 81, 140407 (2010).

${ }^{5}$ S. Sugimoto, M. C. Rosamond, E. H. Linfield, and C. H. Marrows, Appl. Phys. Lett. 109, 112405 (2016).

${ }^{6}$ J. M. Lee, C. Jang, B.-C. Min, S.-W. Lee, K.-J. Lee, and J. Chang, Nano Lett. 16, 62 (2016).

${ }^{7}$ H. T. Nembach, J. M. Shaw, M. Weiler, E. Jué, and T. J. Silva, Nat. Phys. 11, 825 (2015)

${ }^{8}$ J. Cho, N.-H. Kim, S. Lee, J.-S. Kim, R. Lavrijsen, A. Solignac, Y. Yin, D.-S. Han, N. J. J. van Hoof, H. J. M. Swagten, B. Koopmans, and C.-Y. You, Nat. Commun. 6, 7635 (2015). 
${ }^{9}$ K. Di, V. L. Zhang, H. S. Lim, S. C. Ng, M. H. Kuok, J. Yu, J. Yoon, X. Qiu, and H. Yang, Phys. Rev. Lett. 114, 047201 (2015).

${ }^{10}$ A. Fert, N. Reyren, and V. Cros, Nat. Rev. Mater. 2, 17031 (2017).

${ }^{1}$ K.-S. Ryu, L. Thomas, S.-H. Yang, and S. Parkin, Nat. Nanotechnol. 8, 527 (2013).

${ }^{12}$ S.-G. Je, D.-H. Kim, S.-C. Yoo, B.-C. Min, K.-J. Lee, and S.-B. Choe, Phys. Rev. B 88, 214401 (2013).

${ }^{13}$ D.-S. Han, N.-H. Kim, J.-S. Kim, Y. Yin, J.-W. Koo, J. Cho, S. Lee, M. Kläui, H. J. M. Swagten, B. Koopmans, and C.-Y. You, Nano Lett. 16, 4438 (2016).

${ }^{14}$ J.-H. Moon, S.-M. Seo, K.-J. Lee, K.-W. Kim, J. Ryu, H.-W. Lee, R. D. McMichael, and M. D. Stiles, Phys. Rev. B 88, 184404 (2013).

${ }^{15}$ D. Corts-Ortuo and P. Landeros, J. Phys.: Condens. Matter 25, 156001 (2013).

${ }^{16}$ S. Seki, Y. Okamura, K. Kondou, K. Shibata, M. Kubota, R. Takagi, F. Kagawa, M. Kawasaki, G. Tatara, Y. Otani, and Y. Tokura, Phys. Rev. B 93, 235131 (2016).

${ }^{17} \mathrm{~V}$. Vlaminck and M. Bailleul, Phys. Rev. B 81, 014425 (2010).

${ }^{18}$ D. Pozar, Microwave Engineering, 4th ed. (Wiley, 2011).

${ }^{19}$ This is no longer useful for thicknesses larger than the skin depth.

${ }^{20}$ M. Collet, O. Gladii, M. Evelt, V. Bessonov, L. Soumah, P. Bortolotti, S. O. Demokritov, Y. Henry, V. Cros, M. Bailleul, V. E. Demidov, and A. Anane, Appl. Phys. Lett. 110, 092408 (2017).

${ }^{21}$ We use 4 symmetric- and antisymmetric Lorentzians to fit the curves. We need a fourth curve to properly fit the background for some measurements. We believe there to be a fourth spin wave resonance-the quantization of the $k_{s}$ peak-at higher fields, but we are not able to reliable fit this peak.

${ }^{22}$ B. A. Kalinikos, Sov. Phys. J. 24, 718 (1981).
${ }^{23}$ B. A. Kalinikos and A. N. Slavin, J. Phys. C: Solid State Phys. 19, 7013 (1986). ${ }^{24}$ C. Kittel, Phys. Rev. 110, 1295 (1958).

${ }^{25}$ K. Y. Guslienko, S. O. Demokritov, B. Hillebrands, and A. N. Slavin, Phys. Rev. B 66, 132402 (2002).

${ }^{26}$ K. Y. Guslienko, R. W. Chantrell, and A. N. Slavin, Phys. Rev. B 68, 024422 (2003).

${ }^{27}$ T. Schneider, A. A. Serga, T. Neumann, B. Hillebrands, and M. P. Kostylev, Phys. Rev. B 77, 214411 (2008).

${ }^{28} \mathrm{~A}$ simple way to check this would entail removing the magnetic strip between the antennas only. However, as detailed in the supplementary material, this parasitic coupling is also mediated by the magnetic strip. Therefore, it still remains to be explicitly verified that the parasitic coupling is independent of spin waves being transmitted.

${ }^{29}$ Moving the antennas closer together should remedy (at least part of) this issue. ${ }^{30}$ O. Gladii, D. Halley, Y. Henry, and M. Bailleul, Phys. Rev. B 93, 054430 (2016).

${ }^{31}$ We fitted all resonance fields, similar to the analysis in Fig. 2(b), using $g=2.17$, $M_{\mathrm{s}}=1.44 \mathrm{MA} \mathrm{m}{ }^{-1}$, and $k_{\mathrm{s}, \mathrm{m}}=2.16,6 \mu \mathrm{m}^{-1}$, and the fit finds $M_{\text {eff }}=1.02$ $\pm 0.01 \mathrm{MA} \mathrm{m}^{-1}, w_{\text {eff }}=1.2 \pm 0.1 \mu \mathrm{m}$, and $t=10 \pm 0.6 \mathrm{~nm}$. These values agree well with the data from Fig. 2(b). The fit uncertainties for the quantization and secondary peak are quite high - to avoid getting non-nonsensical uncertainties we therefore normalize the fit uncertainties by $\chi^{2}$.

${ }^{32}$ We use only 1 symmetric and antisymmetric Lorentzian to fit the spectrum. The higher order mode for the alternative design is not fitted. It is visible, but the fits consistently placed the peak at a different location. 